\title{
Potential mortality and cost reduction in adult severe sepsis and septic shock through the use of an innovative fluid delivery device
}

This article was published in the following Dove Press journal: Open Access Emergency Medicine

\author{
Elizabeth A Brooks' \\ Mark Piehl ${ }^{2-4}$ \\ 'Health Economics, TTi Health \\ Research and Economics, Hampstead, \\ MD, USA; ${ }^{2} 410$ Medical, Durham, \\ NC, USA; ${ }^{3}$ Pediatric Critical Care, \\ WakeMed Children's Hospital, Raleigh, \\ NC, USA; ${ }^{4}$ Department of Pediatrics, \\ University of North Carolina Chapel \\ Hill, Chapel Hill, NC, USA
}

Correspondence: Elizabeth A Brooks Health Economics, TTi Health Research and Economics, 4500 Black Rock Rd Suite 20I, Hampstead, MD 21074, USA

$\mathrm{Tel}+\mathrm{I} 8005802990$ ext 20 I

Fax + I88839| 5380

Email ebrooks@transtechint.com
Background: While early fluid resuscitation has been shown to significantly improve health and economic metrics in septic shock, providers are often unable to achieve fluid delivery guidelines using current techniques.

Purpose: To examine expected clinical and economic consequences of more consistent achievement of fluid resuscitation guidelines through use of a novel fluid delivery technology.

Patients and methods: A decision analytic model was developed to compare expected costs and outcomes associated with the standard technique vs a novel, faster technique for rapid fluid resuscitation in adult patients with severe sepsis or septic shock.

Results: Use of an innovative fluid delivery device (LifeFlow) resulted in lower expected mortality compared to standard intravenous fluid delivery methods (reduction of 10 fewer deaths per 500 cases). Compared to standard methods, use of the innovative rapid fluid delivery device also resulted in lower expected hospital costs (US\$1,569,131 cost reduction per 500 cases), a lower required use of mechanical ventilation ( $24 \%$ vs $31 \%$ ), decreased average length of stay (11 vs 13 days), decreased average intensive care unit length of stay (2 vs 3 days), and decreased use of vasopressors ( $17 \%$ vs $21 \%$ ). A sensitivity analysis showed that utilization of the rapid fluid delivery device is more cost-effective than standard methods, even under the most conservative assumptions. Conclusion: Based on existing data supporting the importance of early, controlled fluid resuscitation in septic shock patients, the analytical model developed in this study demonstrated the benefit of a novel device that facilitates earlier fluid bolus completion and better adherence to sepsis bundles. Keywords: sepsis syndrome, sepsis bundles, fluid administration, surviving sepsis campaign, resuscitation

\section{Introduction}

Early fluid resuscitation targeted at reversal of shock has been shown to significantly improve mortality, length of hospital stay, and cost in patients with septic shock (SS). ${ }^{1,2}$ However, numerous barriers to effective fluid delivery exist, including poor understanding of guidelines, fear of the adverse effects of fluid overload, and inherent limitations of current fluid delivery techniques. As a result, clinicians often do not achieve published fluid delivery guidelines. ${ }^{3-5}$

The majority of SS patients are fluid-responders and Surviving Sepsis Campaign (SSC) guidelines have specified a 3-hour target time window for initial fluid resuscitation. ${ }^{6}$ There is strong evidence that bundle compliance and adherence to time-sensitive elements of sepsis treatment, including early fluid management, improves patient outcomes. ${ }^{1,4,6-13}$ However, there is no evidence that 3 hours is the optimal resuscitation window. Three hours may not be fast enough in some patients since even short delays in bundle initiation 
can negatively impact outcomes. ${ }^{14}$ The recently proposed SSC Hour-1 bundle ${ }^{15}$ and the surrounding debate highlight the need for more rigorous additional data before developing and implementing broad new clinical guidelines as well as the critical role early fluids can have in some patients. One recent study has demonstrated that initiation of fluid resuscitation in the first 30 minutes of care improves mortality in SS patients. ${ }^{16}$

Current methods of fluid resuscitation include gravity infusion, intravenous (IV) infusion pumps, pressure bags (PBs), manual syringes (the "push-pull" technique, or PPT), and mechanical rapid infusers such as the Level $1^{\circledR}$ and Belmont ${ }^{\circledR}$ Rapid Infuser. All of these techniques have drawbacks that limit the ability of providers to achieve early fluid delivery in SS. Infusion pumps deliver a maximum rate of $1,000 \mathrm{~mL} / \mathrm{hr}$, gravity flow is similarly slow, and while the addition of a pressure bag may speed up the infusion, this technique requires constant re-inflation to achieve adequate flow. With PPT, providers may be able to achieve the bundle guidelines, but this complex and labor-intensive technique may be associated with increased risk of nosocomial infection and is rarely used with adult patients. ${ }^{17,18}$ Mechanical rapid infusers are typically only available in large trauma centers, require significant training for proper use, work best with large-bore IV access, and are frequently unavailable in the hospital outside of the emergency department. ${ }^{19}$

A novel, hand-operated device (LifeFlow ${ }^{\circledR} ; 410$ Medical, Durham, NC, USA) is now available that enables health care providers to rapidly deliver IV fluids to critically ill patients. Simulation and post-market clinical analyses comparing this novel device to other fluid delivery alternatives, specifically pressure bags and PPT, have demonstrated that this new device provides fluid volumes faster than current techniques, likely enabling routine achievement of bundle guidelines. In one such study, clinicians using the novel device were able to complete a SS clinical scenario in approximately half the time as clinicians using pressure bags. ${ }^{20}$ The novel device has also been shown to improve the control and accuracy of fluid bolus size administered compared to standard techniques. ${ }^{20}$

Because adherence to fluid delivery guidelines is associated with improved health outcomes in adult SS, it is important for health care facilities to understand the clinical and economic implications associated with new rapid fluid delivery methods compared to standard techniques. This analysis examines expected clinical and economic consequences which may be associated with more consistent achievement of fluid resuscitation guidelines through the use of a novel, more rapid fluid delivery technique in adult SS.

\section{Materials and methods}

A decision analytic model (Figure 1) was developed to compare expected costs and outcomes associated with the standard techniques vs a novel, faster technique for rapid fluid resuscitation in adult patients with severe sepsis or SS. The analysis was conducted from the perspective of a facility operating within a traditional fee-for-service reimbursement environment where the facility is not accountable for reducing longer-term costs of patient care.

The model relies primarily on the findings of Leisman et al which describe the costs and outcomes associated with patients who are managed in compliance vs in noncompliance with established sepsis bundles for patients presenting with SS. ${ }^{1}$ The Leisman study was utilized as the primary study informing the model for the following reasons: 1) it provided consistent data collection and outcomes from three separate facilities and patient cohorts; 2) it provided the most comprehensive information regarding costs and outcomes associated with patients managed in compliance vs non-compliance with established sepsis bundles; and 3) the publication included data regarding the percentage of time that non-compliance with fluid delivery was the only

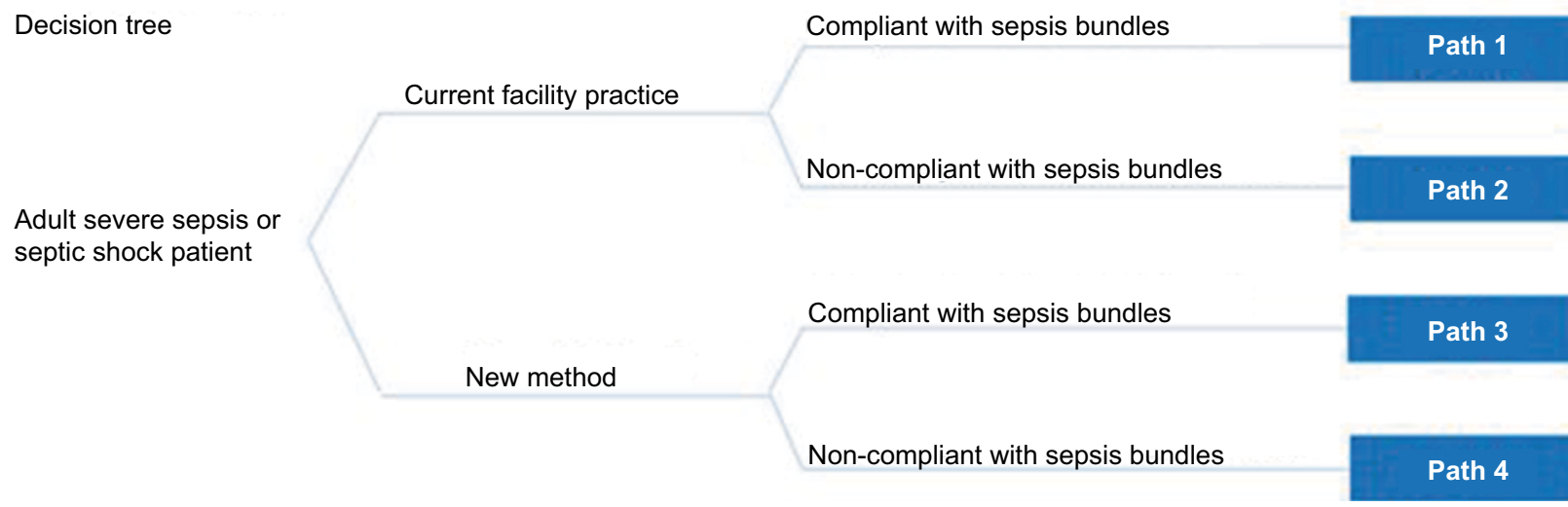

Figure I Decision tree model structure. 
component preventing compliance with the sepsis bundles, enabling estimation of the impact of improved fluid resuscitation on compliance with the bundle as a whole.

The model examined probable outcomes and costs based on existing literature on adult patients with $\mathrm{SS},{ }^{7-10}$ covering the time from presentation to hospital discharge or death (but not subsequent phases of care). Patient mortality, hospital length of stay (LOS), the occurrence of bundle compliant fluid delivery (30 mL/kg within 3 hours), intensive care unit (ICU) LOS, use of vasopressors, mechanical ventilation and facility costs associated with SS diagnosis, vascular access, and IV fluid delivery were tracked. Paths 1 and 2 modeled patients treated with standard methods. Paths 3 and 4 modeled patients treated in an identical manner but assuming a novel, faster technique was used for fluid resuscitation. Paths 1 and 3 model those patients who receive protocol compliant care, whereas Paths 2 and 4 model those patients who receive protocol non-compliant care. It was conservatively assumed that the availability of the novel, faster technique would not influence the timeliness of SS diagnosis or the timeliness of vascular access.

Movement through the decision tree was governed by the likelihood of sepsis bundle compliance. For standard techniques (eg, pressure bag; Paths 1 and 2), transition probabilities dictating patient movement through the model were drawn from the peer-reviewed literature. ${ }^{1,5}$ For the novel fluid delivery technique (Paths 3 and 4), transition probabilities were assigned based on data collected in a single-center study. ${ }^{20}$ Briefly, four critical care clinicians, each with more than 5 years of experience, participated in the 2-day study. All clinicians had prior experience with the novel fluid delivery technology as well as with PPT and PB fluid delivery methods. In each case, the participant was asked to follow hospital or manufacturer protocol to set up and deliver $1 \mathrm{~L}$ of saline as quickly as possible to a simulated patient using each of the three different fluid delivery methods. Setup and infusion times were recorded for each infusion method and corresponding IV gauge. Flow rates of the three techniques were compared using ANOVA (Table S1). With 18-gauge catheters, a size commonly used in adult patients, the novel technology was two-fold faster than pressure bags for delivering a $1 \mathrm{~L}$ fluid bolus; these data informed our base case model assumptions of more favorable bundle compliance for the novel technology.

Model assumptions (Table 1) related to patient mortality and facility costs incurred for compliance vs non-compliance with sepsis bundles were drawn from peer-reviewed literature. ${ }^{1,21}$ All costs were updated to 2017 USD utilizing the Consumer Price Index for health care services. Costs associated with analogous standard methods and new method pathways (eg, Path 1 and Path 3; Path 2 and Path 4) were assumed to be identical except for the additional cost of the new method, estimated to be US $\$ 250$ per patient. Otherwise, the cost of care on each analogous model pathway was similar for patients managed with standard IV fluid delivery methods vs the new method. Mortality rates for standard IV fluid delivery methods vs the new method were identical across analogous model pathways; ie, mortality depends on timesensitive patient outcomes (ie, sepsis diagnosis, vascular access, IV fluid receipt), but not on the means to achieve those outcomes.

In order to demonstrate the robustness of the results, a series of one-way sensitivity analyses were performed using TreeAge Pro (TreeAge Software, Inc., Williamstown, MA, USA). One-way sensitivity analyses consisted of the manipulation of key variables one at a time by increasing or decreasing values across a range that is supported from the peer-reviewed literature, or by $30 \%$ for cost estimates (per standard health economics practice), to determine the impact these changes may have upon the cost-effectiveness findings. Additionally, we conducted a "worst case" sensitivity

Table I Costs and outcome assumptions for bundle compliance vs non-compliance

\begin{tabular}{ll}
\hline & $\begin{array}{l}\text { Bundle compliant } \\
\text { (Paths I and 3) }\end{array}$ \\
\hline Standard method compliance rate & $26 \%$ \\
New method compliance rate & $75 \%$ \\
Total direct mean hospital costs & $\$ 29,363$ \\
Average daily hospital costs & $\$ 2,669$ \\
Probability of mechanical ventilation & $25 \%$ \\
Probability of patient mortality & $\$ 36,808$ \\
Probability of intensive care unit admission & $\$ 2,83$ I \\
Average intensive care unit length of stay (days) & $40 \%$ \\
Average hospital length of stay (days) & 2 \\
Probability of vasopressor use & $11 \%$ \\
Additional per patient new method cost & $17 \%$ \\
\hline Note: All cost are & $\$ 23 \%$ \\
\hline
\end{tabular}

Note: All costs are expressed in US\$. 
analysis in which the least favorable value (with respect to LifeFlow) was adopted for each variable. The findings from this analysis would determine if a new, faster fluid resuscitation method would be cost-effective even under the least favorable conditions.

\section{Results}

The decision to utilize a new, faster fluid delivery method resulted in decreased costs when compared to standard fluid delivery methods $(\$ 1,569,131$ cost reduction per 500 patients) as well as decreased mortality (10 fewer patient deaths per 500 patients). Additionally, as shown in Table 2 , the use of a new method resulted in reductions in total hospital days, number of ICU admissions, number of ICU days, number of patients requiring vasopressors, and number of patients requiring mechanical ventilation.

To determine if model findings were robust to reasonable changes in assumptions, one-way sensitivity and threshold analyses were performed. In all sensitivity analyses, a new, faster fluid delivery method was associated with improved

Table 2 Expected benefits of new, faster fluid delivery method per 500 patients

\begin{tabular}{ll}
\hline Variable & $\begin{array}{l}\text { Expected } \\
\text { benefit }\end{array}$ \\
\hline $\begin{array}{ll}\text { Reduction in expected facility costs } \\
\text { Reduction in patient deaths }\end{array}$ & 10 \\
$\begin{array}{l}\text { Reduction in total hospital days } \\
\text { Reduction in number of patients admitted to intensive }\end{array}$ & 5 \\
$\begin{array}{l}\text { care unit } \\
\text { Reduction in total intensive care unit days } \\
\text { Reduction in number of patients requiring }\end{array}$ & 121 \\
$\begin{array}{l}\text { vasopressors } \\
\text { Reduction in number of patients requiring mechanical } \\
\text { ventilation }\end{array}$ & $\mathrm{II}$ \\
\hline
\end{tabular}

outcomes (including reduced mortality) and lower costs as compared to standard fluid delivery procedures, demonstrating the stability of modeled results (Table 3 ). Additionally, we conducted a "worst case" sensitivity analysis in which the least favorable value (with respect to a new fluid delivery method) was adopted for each variable. The findings from this analysis confirmed that utilization of a new, faster fluid resuscitation method would be cost-effective even under the most conservative assumptions.

\section{Conclusion}

Early fluid resuscitation has been demonstrated to have many significant benefits in the treatment of adult SS. 1,3,9,10,13,16 However, standard fluid delivery techniques have drawbacks that limit the ability of providers to consistently achieve fluid resuscitation goals. The ideal fluid delivery technique would enable more rapid diagnosis and treatment, and would potentially allow clinicians to intervene earlier in the disease process. An innovative, rapid fluid delivery device may offer clinicians greater control and speed in resuscitating critically ill patients. The purpose of this analysis was to evaluate, through an analytical model, the impact of a new, more rapid fluid resuscitation technique on overall bundle compliance and ultimately on patient and economic outcomes.

Our model indicated that the use of a more rapid fluid resuscitation technique may offer a significant cost benefit and mortality reduction compared to standard fluid delivery methods for adult SS presenting in the hospital. Cost savings are primarily due to the shorter inpatient hospital stay, reduced ICU admissions, and other associated costs influenced by increased protocol compliance associated with early, rapid fluid delivery.

Additionally, for the range of values investigated and across all parameters allowed to vary, the new fluid delivery

Table 3 One-way sensitivity analysis

\begin{tabular}{|c|c|c|c|c|}
\hline Model variable & Range & $\begin{array}{l}\text { Base case } \\
\text { assumption }\end{array}$ & $\begin{array}{l}\text { Conservative } \\
\text { assumption }\end{array}$ & $\begin{array}{l}\text { New method cost } \\
\text { saving with conservative } \\
\text { assumption? }\end{array}$ \\
\hline Average LOS for patient not receiving bundle compliant care & $8-18$ days & 13 days & 8 days & Yes \\
\hline $\begin{array}{l}\text { Average reduction in LOS for patient receiving bundle compliant } \\
\text { care }\end{array}$ & I-4 days & 2 days & I day & Yes \\
\hline $\begin{array}{l}\text { Under current practices, \% of time facility achieves bundle } \\
\text { compliant care }\end{array}$ & $26 \%-53 \%$ & $26 \%$ & $53 \%$ & Yes \\
\hline $\begin{array}{l}\% \text { of time that non-compliance with fluid delivery is the cause of } \\
\text { bundle non-compliant care }\end{array}$ & $65 \%-98 \%$ & $82 \%$ & $65 \%$ & Yes \\
\hline $\begin{array}{l}\% \text { of time that new method use will change bundle non- } \\
\text { compliant fluid delivery to bundle compliant }\end{array}$ & $50 \%-90 \%$ & $75 \%$ & $50 \%$ & Yes \\
\hline Mean per-patient cost savings enabled by bundle compliant care & $\$ 6,328-\$ 8,562$ & $\$ 7,445$ & $\$ 6,328$ & Yes \\
\hline
\end{tabular}

Note: All costs are expressed in US\$.

Abbreviation: LOS, length of stay. 
method was the most cost-effective fluid delivery method, even modeling the most conservative assumptions, proving the robustness of the model results. This analysis adds to the knowledge base regarding the outcomes and related facility costs of alternative fluid resuscitation treatments in SS. When compared to standard fluid delivery methods, this new fluid delivery method offers faster, more efficient, and more controlled fluid resuscitation resulting in improved patient health and economic outcomes vs standard fluid delivery methods. Finally, costs included in this model are from the index SS hospitalization only and do not include costs of subsequent re-admissions. This likely underestimates the cost benefits of improved fluid resuscitation, as poor health outcomes associated with bundle non-compliance are likely to result in increased re-admission rates.

\section{Acknowledgment}

Funding for the study was provided by 410 Medical.

\section{Disclosure}

Dr. Piehl is co-founder and Chief Medical Officer of 410 Medical. The authors report no other conflicts of interest in this work.

\section{References}

1. Leisman DE, Doerfler ME, Ward MF, et al. Survival benefit and cost savings from compliance with a simplified 3-Hour sepsis bundle in a series of prospective, multisite, observational cohorts. Crit Care Med. 2017.

2. Rivers E, Nguyen B, Havstad S, et al. Early goal-directed therapy in the treatment of severe sepsis and septic shock. $N$ Engl J Med. 2001;345(19):1368-1377.

3. Leisman DE, Doerfler ME, Schneider SM, Masick KD, D'Amore JA, D'Angelo JK. Predictors, Prevalence, and Outcomes of Early Crystalloid Responsiveness Among Initially Hypotensive Patients With Sepsis and Septic Shock. Crit Care Med. 2018;46(2):189-198.

4. Rhodes A, Evans LE, Alhazzani W, et al. Surviving Sepsis Campaign: International Guidelines for Management of Sepsis and Septic Shock: 2016. Crit Care Med. 2017;45.

5. National Quality Forum. NQF-Endorsed Measures for Infectious Disease 2016-2017. 2017.
6. BurrellAR, McLaws ML, Fullick M, Sullivan RB, Sindhusake D. SEPSIS KILLS: early intervention saves lives. Med JAust. 2016;204(2):1.e1-e7.

7. Grek A, Booth S, Festic E, et al. Sepsis and shock response team: impact of a multidisciplinary approach to implementing Surviving Sepsis Campaign guidelines and surviving the process. Am J Med Qual. 2017;32(5):500-507.

8. Lee SJ, Ramar K, Park JG, Gajic O, Li G, Kashyap R. Increased fluid administration in the first three hours of sepsis resuscitation is associated with reduced mortality: a retrospective cohort study. Chest. 2014;146(4):908-915.

9. Leisman D, Wie B, Doerfler M, et al. Association of Fluid Resuscitation Initiation Within 30 Minutes of Severe Sepsis and Septic Shock Recognition With Reduced Mortality and Length of Stay. Ann Emerg Med. 2016;68(3):298-311.

10. Levy MM, Rhodes A, Phillips GS, et al. Surviving Sepsis Campaign: association between performance metrics and outcomes in a 7.5-year study. Intensive Care Med. 2014;40(11):1623-1633.

11. Liu VX, Morehouse JW, Marelich GP, et al. Multicenter implementation of a treatment bundle for patients with sepsis and intermediate lactate values. Am J Respir Crit Care Med. 2016;193(11):1264-1270.

12. Schorr CA. The Future of Sepsis Care is Now. PPT Presentation. Available from: http://blogs.uw.edu/sepsis/files/2017/03/The-Futureof-Sepsis-Care-is-Now-Schorr.pdf. Accessed August 15, 2018.

13. Seymour CW, Cooke CR, Heckbert SR, et al. Prehospital intravenous access and fluid resuscitation in severe sepsis: an observational cohort study. Crit Care. 2014;18(5):533.

14. Pruinelli L, Westra BL, Yadav P, et al. Delay within the 3-hour Surviving Sepsis Campaign guideline on mortality for patients with severe sepsis and septic shock. Crit Care Med. 2018;46(4):1-505.

15. Levy MM, Evans LE, Rhodes A. The Surviving Sepsis Campaign Bundle: 2018 Update. Crit Care Med. 2018;46(6):997-1000.

16. Leisman DE, Goldman C, Doerfler ME, et al. Patterns and outcomes associated with timeliness of initial crystalloid resuscitation in a prospective sepsis and septic shock cohort. Crit Care Med. 2017;45(10):1596-1606.

17. Bertoglio S, Rezzo R, Merlo FD, et al. Pre-filled normal saline syringes to reduce totally implantable venous access device-associated bloodstream infection: a single institution pilot study. J Hosp Infect. 2013;84(1):85-88.

18. Heid F, Bender C, Gervais H, Schmeck J, Kohnen W, Noppens R. Microbial contamination of anesthetic syringes in relation to different handling habits. Am J Infect Control. 2016;44(3):e15-e17.

19. Subramanyam R, Adler E, Kurth CD, Schultz L, Varughese A. Evaluation of a training model for proficiency with Belmont Rapid Infuser. Poster presentation at the Society for Pediatric Anesthesia, March 2014. Available from: http:/www2.pedsanesthesia.org/meetings/2014winter/ posters/uploads/441-ET-38.pdf. Accessed August 15, 2018.

20. Piehl M, Spangler H, Robertson G, Chenet K. A Novel Technique for Improving Fluid Resuscitation in Septic Shock. Abstract 383. Ann Emerg Med. 2017;70(4):S150.

21. Chalupka AN, Talmor D. The economics of sepsis. Crit Care Clin. 2012;28(1):57-76. 


\section{Supplementary material}

Table SI Fluid delivery flow rates

\begin{tabular}{|c|c|c|c|c|}
\hline \multirow[t]{2}{*}{ Gauge } & \multicolumn{3}{|l|}{$\mathrm{mL} / \mathrm{min}$} & \multirow[t]{2}{*}{$P$-value } \\
\hline & $\begin{array}{l}\text { Pressure } \\
\text { bag }\end{array}$ & Push-pull & $\begin{array}{l}\text { New } \\
\text { method }\end{array}$ & \\
\hline 16 & 120 & 125 & 218 & 0.003 \\
\hline 18 & 96 & 110 & 192 & $<0.001$ \\
\hline 20 & 80 & 101 & 223 & $<0.001$ \\
\hline 22 & 46 & 84 & 193 & $<0.001$ \\
\hline CVC & 47 & 72 & 126 & $<0.001$ \\
\hline
\end{tabular}

Abbreviation: CVC, central venous catheter.

\section{Publish your work in this journal}

The Open Access Emergency Medicine is an international, peerreviewed, open access journal publishing original research, reports, editorials, reviews and commentaries on all aspects of emergency medicine. The manuscript management system is completely online and includes a very quick and fair peer-review system, which is all easy to use. Visit http://www.dovepress.com/testimonials.php to read real quotes from published authors. 